\title{
Methylprednisolone Increases Neuronal Apoptosis during Autoimmune CNS Inflammation by Inhibition of an Endogenous Neuroprotective Pathway
}

\author{
Ricarda Diem, ${ }^{1}$ Muriel Hobom, ${ }^{1}$ Katharina Maier, ${ }^{1}$ Robert Weissert, ${ }^{2}$ Maria K. Storch, ${ }^{3}$ Roman Meyer, ${ }^{2}$ and \\ Mathias Bähr ${ }^{1}$ \\ ${ }^{1}$ Neurologische Universitätsklinik, 37075 Göttingen, Germany, ${ }^{2}$ Neurologische Universitätsklinik, 72076 Tübingen, Germany, and ${ }^{3}$ Neurologische \\ Universitätsklinik, 8036 Graz, Austria
}

\begin{abstract}
Optic neuritis is one of the most common clinical manifestations of multiple sclerosis (MS), a chronic inflammatory disease of the CNS. High-dosage methylprednisolone treatment has been established as the standard therapy of acute inflammation of the optic nerve (ON). The rationale for corticosteroid treatment lies in the antiinflammatory and immunosuppressive properties of these drugs, as shown in experimental autoimmune encephalomyelitis (EAE), the animal model of MS. To investigate the influence of methylprednisolone therapy on the survival of retinal ganglion cells (RGCs), the neurons that form the axons of the $\mathrm{ON}$, we used a rat model of myelin oligodendrocyte glycoprotein (MOG)-induced EAE. Optic neuritis was diagnosed by recording visual evoked potentials, and RGC function was monitored by measuring electroretinograms. Methylprednisolone treatment significantly increased RGC apoptosis during MOG-EAE. By Western blot analysis, we identified the underlying molecular mechanism: a suppression of mitogen-activated protein kinase (MAPK) phosphorylation, which is a key event in an endogenous neuroprotective pathway. The methylprednisolone-induced inhibition of MAPK phosphorylation was calcium dependent. Hence, we provide evidence for negative effects of steroid treatment on neuronal survival during chronic inflammatory autoimmune disease of the CNS, which should result in a reevaluation of the current therapy regimen.
\end{abstract}

Key words: experimental autoimmune encephalomyelitis; glucocorticosteroids; retinal ganglion cells; visual evoked potentials; electroretinogram; mitogen-activated kinases

\section{Introduction}

Glucocorticosteroid pulse therapy is the standard approach for exacerbations of chronic inflammatory autoimmune CNS diseases including acute optic neuritis (Brusaferri and Candelise, 2000). Steroid treatment controls CNS inflammation by inducing T-cell apoptosis (Schmidt et al., 2000; Leussink et al., 2001), modulating the expression of cytokines (Wandinger et al., 1998) or inhibiting leukocyte migration (Gelati et al., 2002). According to the traditional understanding of steroid actions, glucocorticosteroids influence gene expression by interaction with intracellular receptors, which act as ligand-dependent transcription factors. This ligand-dependent modulation of transcription has been termed "genomic" steroid action (Falkenstein et al., 2000) and is sensitive to classical inhibitors of the cytosolic glucocorticoid receptor. For interaction with this receptor, low concentrations of steroids are sufficient, as achieved under low-dose corticosteroid treatment or by endogenous hormone production

Received March 31, 2003; revised May 13, 2003; accepted June 10, 2003.

This work was supported by the Gemeinnützige Hertie Stiftung and the Frauenförderprogramm of the University of Göttingen (Göttingen, Germany). We thank B. Kramer and I. Boger for expert technical assistance. We also thank $G$. Dietz and F. Staub for critically reading this manuscript.

Correspondence should be addressed to Dr. Ricarda Diem, Neurologische Universitätsklinik, Robert-Koch-Strasse 40, D-37075 Göttingen, Germany. E-mail: rdiem@gwdg.de.

R. Meyer's present address: Profos GmbH, 93040 Regensburg, Germany.

Copyright $\odot 2003$ Society for Neuroscience $\quad 0270-6474 / 03 / 236993-08 \$ 15.00 / 0$
(Gold et al., 2001). From recent experimental data, it became evident that high-dose glucocorticosteroid treatment, as used in the therapy of neurological autoimmune disorders, results in effects distinct from genomic steroid action. These so-called "nongenomic," rapid steroid effects are mediated through interaction with biological membranes either via nonclassical membrane receptors or via a direct physicochemical interaction (Gold et al., 2001). It has been shown that nongenomic effects on cellular functions involve modulation of neurotransmission in the rat hippocampus in vivo (Venero and Borrell, 1999) or affect calcium influx into synaptic plasma membranes in vitro (Sze and Iqbal, 1994). A nongenomic steroid-induced regulation of mitogenactivated protein kinases (MAPKs), an endogenous neuroprotective pathway, was demonstrated in a neuroblastoma cell line (Watters et al., 1997), but the in vivo relevance of this effect remained unclear.

From the kinetics of lymphocyte cell death and the effectiveness of high glucocorticosteroid dosages, it has been concluded that the therapeutic induction of T-cell apoptosis during acute autoimmune CNS inflammation might also be a nongenomic phenomenon (Schmidt et al., 2000). Apoptotic cell death induced by corticosteroids as an unwanted side effect of steroid treatment during chronic inflammatory diseases has been described for chondrocytes (Nakazawa et al., 2002) or epithelial cells (Dorscheid et al., 2001). The vulnerability of hippocampal 
neurons to glucocorticosteroid action has been classified as an exception when compared with other neuronal cell types (Almeida et al., 2000), explained by the enriched density of glucocorticoid receptors in subregions of the hippocampus (De Kloet et al., 1998).

Experimental autoimmune encephalomyelitis (EAE), the animal model of multiple sclerosis (MS), induced by immunization with recombinant rat myelin oligodendrocyte glycoprotein $\left(\right.$ rrMOG $^{\text {Igd }}$ ) affects the optic nerve in $80-90 \%$ of female brown Norway (BN) rats (Weissert et al., 1998; Meyer et al., 2001). In the present study, we investigated the effects of high-dosage methylprednisolone therapy on the survival and function of retinal ganglion cells (RGCs), the neurons that form the axons of the optic nerve $(\mathrm{ON})$, in animals suffering from severe optic neuritis. Optic neuritis was diagnosed by recording visual evoked potentials (VEPs). RGC function was monitored by measuring electroretinograms (ERGs). Moreover, we studied the methylprednisolonedependent downstream signaling cascade that leads to inhibition of MAPK phosphorylation and neuronal apoptosis in vivo.

\section{Materials and Methods}

Rats. Female BN rats (8-10 weeks of age) were used in all of the experiments. They were obtained from Charles River (Sulzfeld, Germany) and kept under environmentally controlled conditions without the presence of pathogens.

All of the experiments that involve animal use were performed in compliance with the relevant laws and institutional guidelines. These experiments have been approved by the local authorities of Braunschweig, Germany.

Immunogen. $\mathrm{rrMOG}^{\mathrm{Igd}}$, corresponding to the $\mathrm{N}$-terminal sequence of rat myelin oligodendrocyte glycoprotein (MOG) (amino acids 1-125), was expressed in Escherichia coli and purified to homogeneity by chelate chromatography (Weissert et al., 1998). The purified protein in $6 \mathrm{~m}$ urea was then dialyzed against PBS to obtain a preparation that was stored at $-20^{\circ} \mathrm{C}$.

Induction and evaluation of EAE. The rats were anesthetized by inhalation of anesthesia with methoxyflurane (Metofane; Pitman-Moore, Mundelein, IL) and injected intradermally at the base of the tail with a total volume of $100 \mu \mathrm{l}$ of inoculum containing $50 \mu \mathrm{g}$ of $\mathrm{rrMG}^{\text {Igd }}$ in saline emulsified (1:1) with complete Freund's adjuvant (CFA) (Sigma, St. Louis, MO) containing $200 \mu \mathrm{g}$ of heat-inactivated Mycobacterium tuberculosis (strain H 37 RA; Difco, Detroit, MI). Rats were scored for clinical signs of EAE and weighed daily. The signs were scored as follows: grade 1, tail weakness or tail paralysis; grade 2, hindleg paraparesis or hemiparesis; grade 3, hindleg paralysis or hemiparalysis; and grade 4, complete paralysis (tetraplegy), moribund state, or death (data not shown).

Retrograde labeling of RGCs. Two weeks before immunization was done, adult $\mathrm{BN}$ rats were anesthetized with intraperitoneal chloral hydrate $(0.42 \mathrm{mg} / \mathrm{kg}$ of body weight), the skin was incised mediosagitally, and holes were drilled into the skull above each superior colliculus ( 6.8 $\mathrm{mm}$ dorsal and $2 \mathrm{~mm}$ lateral from bregma). We injected stereotactically 2 $\mu l$ of the fluorescent dye Fluorogold (FG) (5\% in normal saline; Fluorochrome, Englewood, CO) into both superior colliculi.

Electrophysiological recordings. The rats were anesthetized by intraperitoneal injection of $10 \%$ ketamine $(0.65 \mathrm{ml} / \mathrm{kg}$; Atarost, Twistringen, Germany) together with $2 \%$ xylazine $(0.35 \mathrm{ml} / \mathrm{kg}$; Albrecht, Aulendorf, Germany) and mounted on a stereotaxic device. During the experiment, body temperature was maintained between 35 and $37^{\circ} \mathrm{C}$ with a heating pad, and the electrocardiogram was continuously monitored on an oscilloscope. For recording of VEPs from the primary visual cortex, two gold-screw electrodes with a tip diameter of $1 \mathrm{~mm}$ were placed 3-4 mm lateral to the interhemispheric fissure and $1 \mathrm{~mm}$ frontal to the lambda fissure. Reference electrodes were placed $1 \mathrm{~mm}$ lateral to the midline and $1 \mathrm{~mm}$ before bregma. The ERG was recorded with chlorinated silver-ball electrodes as described previously (Meyer et al., 2001). Visual stimuli were presented on a 17 inch monitor (Acer View 76i) positioned $20 \mathrm{~cm}$ in front of the eye. The display was centered in a position $\sim 40^{\circ}$ medially from the pupil axis. Light flashes of $20 \mu \mathrm{sec}$ duration were used at a rate of $1 \mathrm{~Hz}$, and bar stimulation consisted of vertical gratings of variable spatial frequency, alternating in phase with a temporal frequency of 1.8 $\mathrm{Hz}$ at $66 \%$ Michelson contrast (constant mean luminance, $15 \mathrm{~cd} / \mathrm{m}^{2}$ ). Signals were amplified 10,000-fold and bandpass filtered between 0.1 and $100 \mathrm{~Hz}$, and 128 events were averaged to improve the signal-to-noise ratio. Amplitudes of pattern ERG and pattern VEP were determined as described previously (Meyer et al., 2001). Assessment of visual acuity was also described previously (Meyer et al., 2001). VEP and ERG measurements were performed at clinical onset of the disease before the first dose of methylprednisolone or placebo was given. To monitor the disease course and to investigate the therapeutic effects of methylprednisolone, a second measurement of VEPs and ERGs was done on day 8 of the disease.

Treatment of animals. Animals were treated with intraperitoneal injections of methylprednisolone $(20 \mathrm{mg} / \mathrm{kg}$; Urbason; Hoechst Marion Roussel, Frankfurt/Main, Germany) or vehicle $(0.9 \% \mathrm{NaCl})$ on days $1-3$ or 4-6 of the disease. Other animal groups received intraperitoneal injections of mifepristone (RU 486) (10 mg/kg; Biomol Research Laboratories, Plymouth Meeting, PA) alone, or together with methylprednisolone, or intravitreal injections of cobalt chloride $\left(\mathrm{CoCl}_{2}\right)(2 \mu \mathrm{l}$ of a $100 \mathrm{~mm}$ solution; Sigma) alone, or together with intraperitoneally given methylprednisolone on days 1-3 of the disease. To inhibit the activation of MAPKs, an additional animal group was treated with the intravitreally applied MAPK kinase (MEK) inhibitor $2^{\prime}$-amino-3'-methoxyflavone (PD 98059) (2 $\mu \mathrm{l}$ of a $20 \mathrm{~mm}$ solution; Calbiochem, San Diego, CA).

Quantification of RGC density. At the end of the second recording session, the rats received an overdose of chloral hydrate and were perfused via the aorta with $4 \%$ paraformaldehyde in PBS. The brain, the optic nerves, and both eyes were removed, and the retinas were dissected and flat-mounted on glass slides. They were examined by fluorescence microscopy (Axiophot 2; Zeiss, Göttingen, Germany) using an UV filter (365-397 nm), and RGC densities were determined by counting labeled cells in three areas $\left(62,500 \mu \mathrm{m}^{2}\right)$ per retinal quadrant at three different eccentricities of one-sixth, three-sixths, and five-sixths of the retinal radius. Cell counts were performed by two independent investigators following a blind protocol. Statistical significance was assessed using oneway ANOVA followed by Duncan's test.

Immunohistochemistry. Immunostaining was performed with cryostat sections (18 $\mu \mathrm{m}$ thick) of retinas that were prepared $6 \mathrm{hr}$ after the last application of methylprednisolone or normal saline. DNA fragmentation of cells undergoing apoptosis was analyzed by the terminal deoxynucleotidyl transferase-mediated biotinylated UTP nick end labeling (TUNEL) method. Sections were incubated with $50 \mathrm{U}$ of terminal transferase and $1 \mathrm{~mm}$ biotin-dUTP in the presence of $1.5 \mathrm{mM} \mathrm{CoCl}_{2}, 0.2 \mathrm{M}$ $\mathrm{K}^{+}$-cacodylate, and $25 \mathrm{mg} / \mathrm{ml}$ bovine serum albumin for $90 \mathrm{~min}$ at $37^{\circ} \mathrm{C}$. Incorporated biotinylated nucleotides were detected by incubation with fluorescein isothiocyanate-conjugated streptavidin and examined by fluorescence microscopy. TUNEL-positive RGCs were counted by two independent investigators following a blind protocol. For each treatment group, eight retinal sections comparable in size and location were processed. Statistical significance was assessed using Student's $t$ test.

Histopathology. Histological evaluation was performed on paraformaldehyde-fixed, paraffin-embedded sections of brains and spinal cords. Paraffin sections were stained with hematoxylin-eosin, Luxol fast blue, and Bielschowsky silver impregnation to assess inflammation, demyelination, and axonal pathology, respectively, as described previously (Storch et al., 1998) (data not shown). In adjacent serial sections, immunohistochemistry was performed with ED1 antibody (Serotec, Oxford, UK) against macrophage-activated microglia. Bound primary antibody was detected with a biotin-avidin technique. Control sections were incubated in the absence of primary antibody (Storch et al., 1998). The investigators who processed tissue sections and diagnosed optic neuritis were blinded to the electrophysiological and immunohistochemical results of the study.

Western blotting. The Western blot analysis was performed as described previously (Diem et al., 2001). After incubation with the primary antibody against phospho-Akt [New England Biolabs, Schwalbach, Germany; $1: 1000$ in $1 \%$ skim milk in $0.1 \%$ Tween 20 in PBS (PBS-T)], 
membranes were washed in PBS-T and incubated with HRP-conjugated secondary antibodies against rabbit IgG (Santa Cruz Biotechnology, Santa Cruz, CA; 1:2000 in PBS-T). Labeled proteins were detected using the ECL-plus reagent (Amersham Biosciences, Arlington Heights, IL).

For Western blot analysis of B-cell lymphoma-2 (Bcl-2) levels, the primary antibody (sc-7382; Santa Cruz Biotechnology) was diluted 1:200 in 5\% skim milk in PBS-T; for protein detection, an HRP-conjugated secondary antibody against mouse IgG was used (Santa Cruz Biotechnology; 1:2000 in 1\% skim milk in PBS-T).

p44-p42 MAPK protein levels were detected using a primary antibody (sc-93-G; Santa Cruz Biotechnology) diluted 1:500 in 1\% skim milk in PBS-T, and an HRP-conjugated secondary antibody against goat IgG (Santa Cruz Biotechnology; 1:3000 in PBS-T).

For Western blot analysis of phospho-p44-phospho-p42 MAPK levels, the primary antibody ( $\mathrm{Thr}^{180} / \mathrm{Tyr}^{182}$; New England Biolabs) was diluted 1:200 in 1\% skim milk in PBS-T; for protein detection, an HRPconjugated secondary antibody against rabbit IgG was used (Santa Cruz Biotechnology; 1:3000 in PBS-T).

Nitric oxide synthase (NOS) 1 protein levels were detected using a primary antibody (sc-648; Santa Cruz Biotechnology), diluted 1:200 in $5 \%$ skim milk in PBS-T, and an HRP-conjugated secondary antibody against rabbit IgG (Santa Cruz Biotechnology; 1:2000 in 1\% skim milk in PBS-T).

\section{Results}

Methylprednisolone treatment does not improve visual function in rats with severe optic neuritis

To study the influence of high-dose corticosteroid therapy on the survival and function of RGCs during acute optic neuritis, we used a rat model of MOG-induced EAE. Disease onset was at day $15.7 \pm 2.1$ postimmunization (mean \pm SEM). The function of the optic system was investigated by VEP and ERG recordings in response to flash and pattern stimuli. Flash VEP experiments were performed to test axonal signaling of the ON corresponding to the animal's ability to discriminate between light and dark. Pattern VEP and ERG stimuli were used to estimate the animal's visual acuity. Pattern ERG is a specific electrophysiological marker for RGC function, whereas flash ERG represents the function of all of the electrically active cells in the retina (Meyer et al., 2001). Figure $1 a$ gives an example of normal VEP potentials in response to repetitive flash stimuli, whereas Figure $1 b$ shows recordings in an animal with histopathologically proven optic neuritis. Recently, we demonstrated that healthy control CFAimmunized rats have visual acuity values of $1.31 \pm 0.16$ cycles $/^{\circ}$ determined by pattern VEP recordings and $1.10 \pm 0.13$ cycles $/{ }^{\circ}$ in the pattern ERG measurements (Meyer et al., 2001). In the present treatment study, only rats with absent VEP responses to flash and pattern stimulation at day 1 of the disease, indicating severe optic neuritis, were included (Table 1). Each eye was measured separately. Most of the animals (11 of 16 tested eyes) showed clear responses to flash ERG stimulation, demonstrating intact function of the entire retina. None of the animals showed a response to ERG pattern stimulation at day 1 of the disease when the first electrophysiological assessment was performed (Table 1).

To investigate the effects of high-dose methylprednisolone treatment on electrophysiological functions of the optic system, animals were given methylprednisolone intraperitoneally on days 1-3 or 4-6 of the disease. Each group consisted of eight tested eyes. The dosage of $20 \mathrm{mg} / \mathrm{kg}$ of body weight was comparable with the one administered during methylprednisolone pulse therapy of patients with MS (Beck et al., 1992). On day 8 of the disease, when the second electrophysiological assessment was performed, only two of eight tested eyes in the early treatment group and none of the eight eyes in the late treatment group a

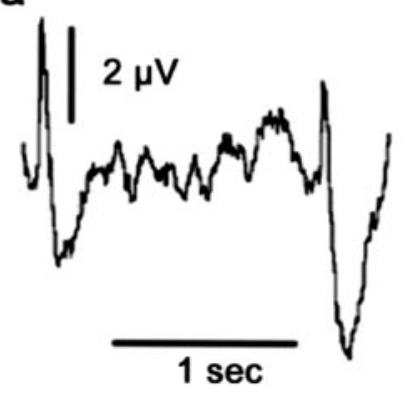

b
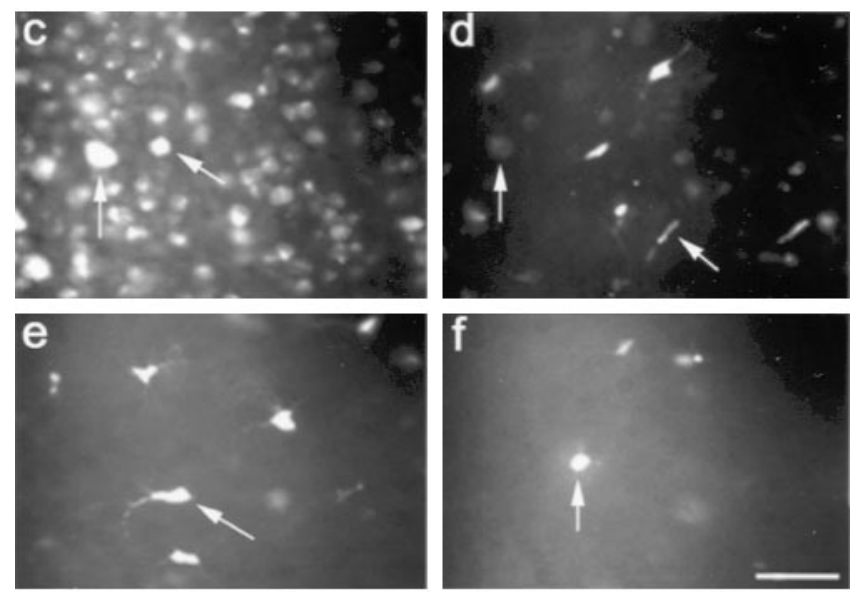

Figure 1. Methylprednisolone decreases RGC survival in rats with electrophysiologically diagnosed optic neuritis. $a$, Example of two normal VEP potentials (at the beginning and the end of the presented recording sequence) in response to repetitive flash stimulation. $b$, Flash VEP recording in an animal with histopathologically proven optic neuritis. Flash stimuli were again applied at the beginning and the end of the presented recording sequence. Only background noise levels of electrical activity were detectable. c, Representative whole-mount area at threesixths retinal radius from a CFA-immunized control rat. Examples of FG-labeled RGCs are indicated by arrows. $d$, The number of FG-labeled RGCs in a vehicle-treated animal with manifest optic neuritis (day 8 of the disease) is significantly decreased. Remaining RGCs appear pale (left arrow). Single FG-labeled endothelial cells are detectable (right arrow). e, An additional significant reduction of RGC density (day 8 of the disease) occurred under treatment with methylprednisolone (days 1-3). Note the predominance of cells with irregularly sized and ramificated dendritic processes corresponding to microglia (arrow). f, Only very few FG-labeled remaining RGCs (arrow) are detectable after treatment with methylprednisolone on days 4-6 of the disease. Again, a representative whole-mount area at three-sixths retinal radius from day 8 of EAE is shown. Scale bar, $100 \mu \mathrm{m}$.

regained a response to flash VEP stimulation. No VEP response to pattern stimulation was detectable in both of the groups after methylprednisolone treatment. These results were comparable with those of the control groups of animals with severe optic neuritis and same electrophysiological inclusion criteria (absent VEP response to flash and pattern stimulation at disease manifestation) (Table 1). These control animals were treated with intraperitoneally given injections of physiological salt solution on days $1-3$ or $4-6$ of the disease and received the second electrophysiological assessment on day 8 of EAE. In the early-treatment control group, three of eight tested eyes and in the late-treatment control group, two of eight tested eyes regained a potential in response to flash VEP stimulation. None of the eyes showed a VEP response to pattern stimulation after vehicle treatment $(\mathrm{Ta}-$ ble 1).

Analysis of ERG recordings revealed that methylprednisolone treatment did not improve this electrophysiological parameter either. Only one of eight eyes in the early-treatment group and none of the tested eyes in the late-treatment group regained a 
Table 1. Results of VEP and ERG recordings (obtained on days 1 and 8 of MOG-EAE) before and after treatment with high-dose methylprednisolone (given on days 1-3 or 4- 6 of the disease)

\begin{tabular}{|c|c|c|c|c|c|c|c|c|}
\hline & \multicolumn{2}{|c|}{ VEP (day 1 EAE) } & \multicolumn{2}{|c|}{ VEP (day 8 EAE) } & \multicolumn{2}{|c|}{ ERG (day 1 EAE) } & \multicolumn{2}{|c|}{ ERG (day 8 EAE) } \\
\hline & Flash & $\begin{array}{l}\text { Large } \\
\text { pattern }\end{array}$ & Flash & $\begin{array}{l}\text { Large } \\
\text { pattern }\end{array}$ & Flash & $\begin{array}{l}\text { Large } \\
\text { pattern }\end{array}$ & Flash & $\begin{array}{l}\text { Large } \\
\text { pattern }\end{array}$ \\
\hline Methlyprednisolone (days 1-3) & $0 / 8$ & $0 / 8$ & $2 / 8$ & $0 / 8$ & $3 / 8$ & $0 / 8$ & $6 / 8$ & $1 / 8$ \\
\hline Placebo (days 1-3) & $0 / 8$ & $0 / 8$ & $3 / 8$ & $0 / 8$ & $8 / 8$ & $0 / 8$ & $8 / 8$ & $0 / 8$ \\
\hline \multicolumn{9}{|l|}{ Methylprednisolone (days } \\
\hline $4-6)$ & $0 / 8$ & $0 / 8$ & $0 / 8$ & $0 / 8$ & $8 / 8$ & $0 / 8$ & $8 / 8$ & $0 / 8$ \\
\hline Placebo (days 4-6) & $0 / 8$ & $0 / 8$ & $2 / 8$ & $0 / 8$ & $8 / 8$ & $0 / 8$ & $8 / 8$ & $0 / 8$ \\
\hline \multirow[t]{2}{*}{ Control (CFA immunized) } & $\begin{array}{l}8 / 8 \\
\text { (day } 16\end{array}$ & $\begin{array}{l}8 / 8 \\
\text { (day } 16\end{array}$ & & & $\begin{array}{l}8 / 8 \\
\text { (day } 16\end{array}$ & $\begin{array}{l}8 / 8 \\
\text { (day } 16\end{array}$ & & \\
\hline & p.i.) & p.i.) & & & p.i.) & p.i.) & & \\
\hline
\end{tabular}

Each group contained eight tested eyes. The number of eyes with detectable potentials in response to flash or pattern stimulation is given as $x$ out of eight eyes $(x / 8)$. Electrophysiological inclusion criteria consisted of absent VEP responses to flash and pattern stimulation at disease onset, indicating severe optic neuritis. Control animals immunized with CFA were measured on day 16 postimmunization (p.i.). Large pattern corresponds to stimulation with three alternating bars.

response to large-pattern stimulation (three alternating bars) (Table 1). These results did not differ from those of the control animals: None of the eight tested eyes in each group responded to large-pattern ERG stimulation during the second electrophysiological assessment performed on day 8 of the disease (Table 1).

\section{Treatment with methylprednisolone decreases the number of} surviving RGCs

In control retinas of healthy CFA-immunized rats, mean RGC density was $2730 \pm 145$ cells $/ \mathrm{mm}^{2}$ (mean \pm SEM; $n=9$ ) (Figs. $1 c, 2 a)$, as determined by retrograde labeling with FG from both superior colliculi. Recently, we showed that, during MOG-EAE, a significant reduction of RGC density occurs, and that this early neuronal cell death shows the morphological and intracellular characteristics of apoptosis such as DNA degradation and caspase-3 activation (Meyer et al., 2001). On the basis of our above-given electrophysiological results, showing no detectable functional benefit after high-dose methylprednisolone therapy, we here investigated steroid effects on RGC survival. On day 8 of the disease, both methylprednisolone treatment groups showed a significant reduction of RGC density when compared with vehicle-treated animals. Cell counts of the early methylprednisolone treated group were in the range of $430 \pm 58 \mathrm{cells} / \mathrm{mm}^{2}$ $(n=8 ; p<0.05)$ (Figs. $1 e, 2 a-c)$, whereas vehicle treatment resulted in a survival of $775 \pm 112 \mathrm{RGCs} / \mathrm{mm}^{2}(n=8)$ (Figs. $1 d$, $2 b$ ). The methylprednisolone group treated on days 4-6 after onset of clinical symptoms showed an RGC reduction to $298 \pm$ 35 cells $/ \mathrm{mm}^{2}(n=10 ; p<0.05)$ (Figs. $\left.1 f, 2 a\right)$ compared with $741 \pm 98$ cells $(n=8)$ of the corresponding control group. In both methylprednisolone-treated groups, the amount of TUNEL-positive RGCs was significantly increased when compared with the vehicle-treated animals. The mean density of TUNEL-positive RGCs in the early methylprednisolone treatment group was $8.9 \pm 1.2$ cells/section $(n=8$; mean \pm SEM; $p<$ $0.05)$ compared with $4.1 \pm 0.9$ TUNEL-positive RGCs/section of the corresponding control group $(n=8)$. The animal group that received methylprednisolone from days $4-6$ of the disease showed 7.5 \pm 1.7 TUNEL-positive RGCs/section versus $3.6 \pm 0.5$ cells of the vehicle-treated control group $(n=8$ in each group; $p<0.05)$. TUNEL-positive RGCs were assessed $6 \mathrm{hr}$ after the last application of methylprednisolone or vehicle. Figure 3 shows two representative retinal sections with increased numbers of TUNEL-positive RGCs under early treatment with methylprednisolone $(c, d)$ compared with vehicle $(a, b)$.

\section{Methylprednisolone-induced reduction of RGC survival is} mediated through a nongenomic mechanism

To test whether the proapoptotic effects of methylprednisolone were mediated through a genomic or nongenomic mechanism, animals were treated with a combination of methylprednisolone (20 mg/kg of body weight) and RU 486 (10 mg/kg of body weight), a competitive antagonist of the cytosolic glucocorticoid receptor (Cadepond et al., 1997). On day 8 of the disease, RGC counts under this combined therapy administered intraperitoneally on days $1-3(n=6)$ were similar to those achieved under treatment with methylprednisolone alone $(390 \pm 39$ vs $430 \pm 58$ cells $/ \mathrm{mm}^{2}$ ) (Fig. $2 b$ ), indicating that methylprednisolone acts independent of the activation of the cytosolic glucocorticoid receptor. RU 486 alone $(n=6)$ had no effect on the survival of RGCs when compared with vehicle treatment $(847 \pm 19$ vs $775 \pm 112$ RGCs $/ \mathrm{mm}^{2}$; day 8 of the disease) (Fig. $2 b$ ).

\section{Methylprednisolone does not induce RGC apoptosis in healthy animals}

To test whether EAE is a precondition for steroid-induced RGC death, healthy FG-labeled rats were treated with intraperitoneally given methylprednisolone on days 1-3 in the same concentration as used in EAE animals ( $20 \mathrm{mg} / \mathrm{kg}$ of body weight). On day $14 \mathrm{of}$ the experiment, methylprednisolone-treated retinas showed cell counts of $2655 \pm 59 \mathrm{RGCs} / \mathrm{mm}^{2}(n=8$; mean \pm SEM) (Fig. $2 a)$, which did not differ significantly from control counts of healthy animals treated with physiological salt solution $(2730 \pm 145$ RGCs $/ \mathrm{mm}^{2}$ ) (Fig. 2a).

\section{Methylprednisolone inhibits MAPK phosphorylation}

To further investigate the mechanisms of methylprednisoloneaugmented RGC death, Western blot analysis of potentially involved signal transduction proteins were performed. Previous studies in non-neuronal tissue suggest a possible role for MAPK phosphorylation, the Akt pathway, or NOS activity in steroidrelated signal transduction (for review, see Falkenstein et al., 2000). An involvement of the Bcl-2 family of proteins in glucocorticoid-induced apoptosis has been postulated in a study on hippocampal granule cells (Almeida et al., 2000).

Western blot analysis of retinas was performed under methylprednisolone therapy after early (days 1-3 of the disease) and late (days $4-6$ of the disease) steroid administration $(20 \mathrm{mg} / \mathrm{kg}$ of body weight; $n=4$ for each group). In each group, protein lysates were prepared $6 \mathrm{hr}$ after the third methylprednisolone dosage was 

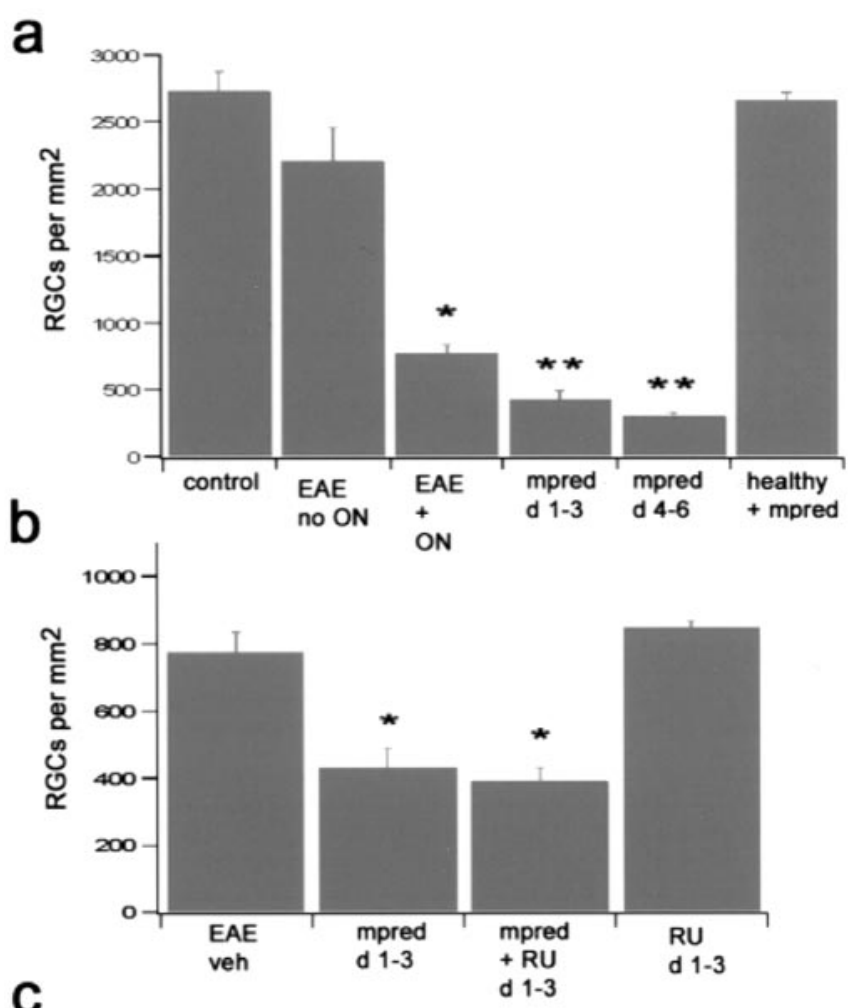

C

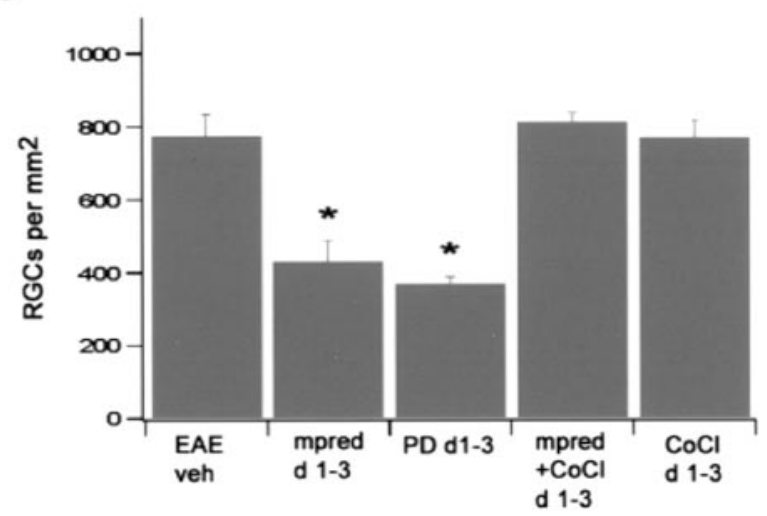

Figure 2. Methylprednisolone decreases RGC survival by a nongenomic, calcium-dependent mechanism. $a$, Data are given as the mean \pm SEM of retrogradely labeled RGCs per square millimeter. The left bar shows RGC counts of control animals immunized with CFA (control) $(n=9)$. No significant reduction of RGC density was detected in animals with manifest EAE but without optic nerve affection (EAE, no $O N)(n=10)$. Animals with manifest optic neuritis showed a significant decrease in RGC counts on day 8 of EAE when compared with controls (EAE $+0 \mathrm{~N})(n=8)$. An additional significant reduction of RGC density was observed after treatment with methylprednisolone (mpred) on days $1-3(n=8)$ or days $4-6(n=10)$ of EAE. Methylprednisolone did not induce RGC death in healthy animals $14 \mathrm{~d}$ after treatment (healthy + mpred) $(n=8) .{ }^{* * * *}$ Statistically significant when compared with controls $\left({ }^{*} p<0.05\right.$; ${ }^{* *} p<$ 0.01 ; one-way ANOVA followed by Duncan's test). $b$, The left bar shows RGC counts on day 8 of MOG-EAE after vehicle treatment with PBS given intraperitoneally on days 1-3 of the disease (EAE veh) $(n=8)$. Combined treatment with RU 468 and methylprednisolone on days $1-3$ of $\mathrm{EAE}$ (mpred $+\mathrm{RU})(n=6)$ did not show any difference in RGC counts when compared with methylprednisolone treatment alone. The right bar gives RGC counts after monotherapy with $\mathrm{RU} 468(\mathrm{RU})(n=6)$. *Statistically significant when compared with EAE veh ( $p<0.05$; oneway ANOVA followed by Duncan's test). c, RGC counts on day 8 of EAE after intravitreal application of vehicle (EAE veh) $(n=6)$ are shown as left bar. Intravitreal injections of PD 98059 (PD) (days $1-3$ of $\mathrm{EAE} ; n=6$ ) resulted in a similar reduction of $\mathrm{RGC}$ density when compared with methylprednisolone treatment. Combined treatment of intraperitoneally given methylprednisolone and intravitreal application of $\mathrm{COCl}_{2}$ (mpred $+\mathrm{CoCl}$ ) (days 1-3 of EAE; $n=6$ ) abolished methylprednisolone-induced decrease in RGC density. The right bar shows RGC counts after treatment with $\mathrm{COCl}_{2}$ alone $(n=6)$. ${ }^{*}$ Statistically significant when compared with EAE veh ( $p<0.05$; one-way ANOVA followed by Duncan's test).
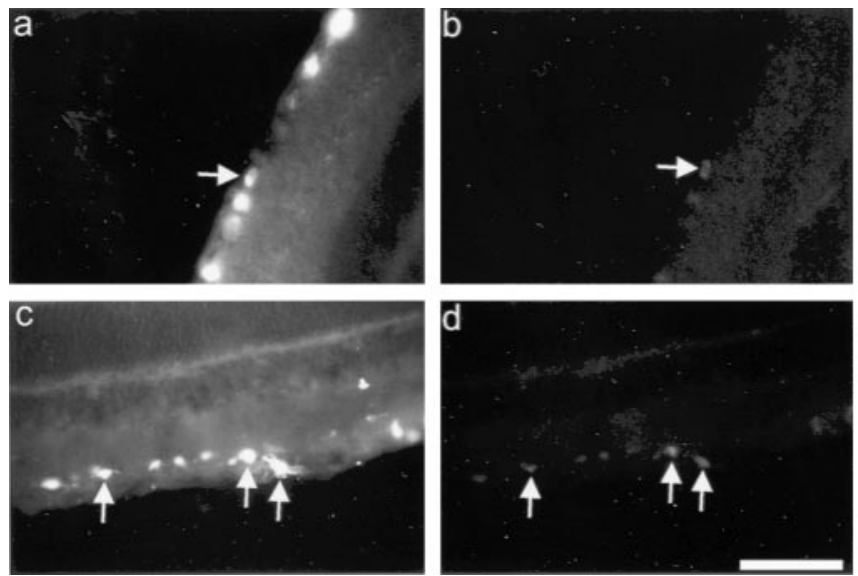

Figure 3. RGC death under methylprednisolone treatment is accompanied with DNA degradation. Examples of cells that show a colocalization for FG $(a, c)$ and TUNEL staining $(b, d)$ representing apoptotic RGCs are indicated by arrows. $a, b$, Double staining of a representative retina section from a $B N$ rat with severe optic neuritis after vehicle treatment. $c, d$, Retina section of a methylprednisolone-treated animal with optic neuritis. The number of TUNEL-positive RGCs is increased under glucocorticoid therapy. Scale bar, $70 \mu \mathrm{m}$.

given. Protein levels of NOS1, phospho-Akt, and Bcl-2 were comparable with those under vehicle treatment with physiological salt solution (Fig. 4a). Analysis of phospho-p44 and phospho-p42 MAPK under steroid therapy revealed a strong downregulation of phospho-p42 MAPK, whereas levels of the unphosphorylated form of both proteins were unchanged (Fig. 4a).

\section{Treatment with an inhibitor of MAPK phosphorylation} mimics the effects of methylprednisolone on RGC survival To test whether the methylprednisolone-induced downregulation of phospho-MAPKs is functionally relevant for the decrease of RGC survival in EAE animals, rats were treated with PD 98059. PD 98059 is a selective and cell-permeable inhibitor of the single upstream kinase MEK, which in turn phosphorylates and thereby activates MAPKs (Kültz et al., 1998). PD 98059 was given intravitreally on days $1-3$ of the disease ( $2 \mu \mathrm{l}$ of a $20 \mathrm{~mm}$ solution; $n=$ 6 ), and its efficiency was verified by Western blot analysis (Fig. $4 b)$. According to the methylprednisolone treatment protocol, RGC densities were analyzed on day 8 of the disease. Treatment with PD 98059 decreased the number of surviving RGCs to a similar extent as did methylprednisolone therapy $(370 \pm 70$ vs $430 \pm 58 \mathrm{RGCs} / \mathrm{mm}^{2}$; mean \pm SEM) (Fig. $2 c$ ). Intravitreal application of vehicle on days $1-3$ of EAE $(n=6)$ had no effect on surviving RGCs $\left(755 \pm 58 \mathrm{RGCs} / \mathrm{mm}^{2}\right.$ ) (Fig. $2 c$ ) when compared with the intraperitoneally treated control group (775 \pm 112 RGCs $/ \mathrm{mm}^{2} ; n=8$ ) (Fig. 2b).

\section{Methylprednisolone-induced enhancement of RGC}

degeneration depends on calcium influx through voltagegated calcium channels

To investigate the involvement of steroid-induced calcium influx in our model, we treated rats with a combination of intraperitoneally given methylprednisolone and intravitreal injections of $\mathrm{CoCl}_{2}(2 \mu \mathrm{l}$ of a $10 \mathrm{~mm}$ solution on days $1-3$ of EAE; $n=6)$, a nonselective blocker of voltage-gated calcium channels (Schenberg et al., 2000). This combined treatment completely abolished the methylprednisolone-induced enhancement of RGC apoptosis ( $812 \pm 26$ vs $755 \pm 58 \mathrm{RGCs} / \mathrm{mm}^{2}$ for vehicle treatment) (Fig. $2 c$ ), whereas cell counts at day 8 of MOG-EAE under treatment with $\mathrm{CoCl}_{2}$ alone did not differ significantly from controls (769 \pm 
a

NOS 1

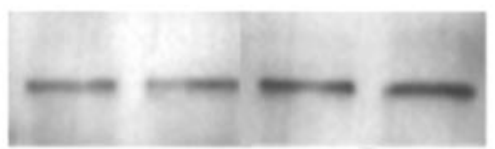

$-160 \mathrm{kDa}$

pAkt
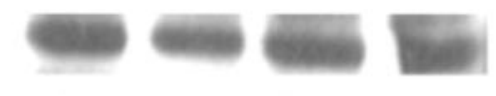

$-60 \mathrm{kDa}$
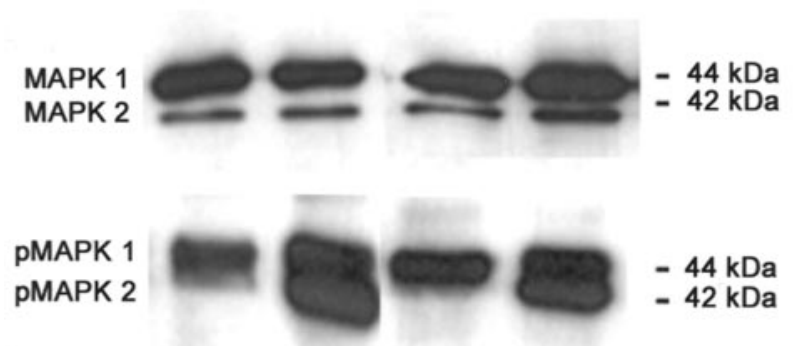

- $44 \mathrm{kDa}$

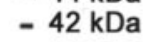

$\mathrm{Bcl}-2$

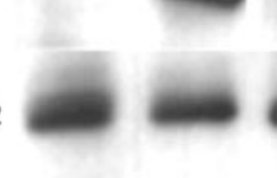
mpred d 1-3 veh
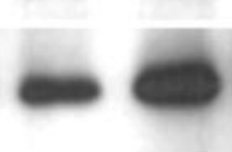

$-23 \mathrm{kDa}$

b

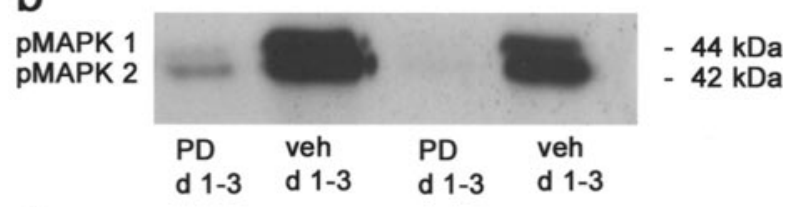

C

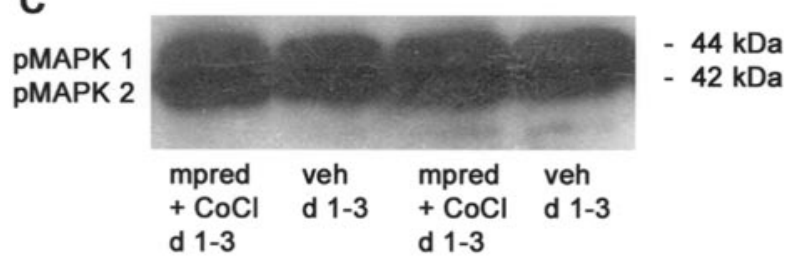

Figure 4. Methylprednisolone inhibits MAPK phosphorylation. $a$, Western blot analysis of NOS1, phospho-Akt (pAkt), MAPK1 and -2, phospho-MAPK (pMAPK) 1 and -2, and Bcl-2 after treatment with methylprednisolone (mpred) on days $1-3$ or $4-6$ of EAE compared with vehicle treatment (veh). Note the strong decrease of the phosphorylated form of MAPK2 under methylprednisolone treatment, whereas levels of the unphosphorylated MAPKs are similar in each group. $b$, Western blot analysis of phospho-MAPKs after intravitreal application of PD 98059 (PD) compared with vehicle controls. c, Western blot analysis of phospho-MAPKs after combined treatment of intraperitoneally given methylprednisolone and intravitreal application of $\mathrm{CoCl}_{2}(\mathrm{CoCl})$ compared with vehicle controls.

46 vs $755 \pm 58$ for vehicle treatment) (Fig. $2 c$ ). The fact that the combined treatment with methylprednisolone and a blocker of voltage-gated calcium channels re-increased RGC counts to control levels raised the hypothesis of a cascade-like order of methylprednisolone-induced calcium influx and inhibition of the MAPK pathway. This was confirmed by Western blot analysis of retinas after cotreatment with methylprednisolone and $\mathrm{CoCl}_{2}$, which showed equal amounts of phosphorylated MAPK protein when compared with vehicle-treated controls (Fig. 4c).

\section{Discussion}

Recently, we demonstrated that severe optic neuritis in animals suffering from MOG-EAE leads to early apoptotic cell death of RGCs (Meyer et al., 2001). In the present study, we investigated the influence of high-dose methylprednisolone treatment, the standard therapy of acute autoimmune optic nerve inflammation (Kaufman et al., 2000), on survival and function of the neurons that form the axons of the ON. As revealed by VEP and ERG recordings, methylprednisolone therapy did not improve visual functions in animals with electrophysiologically diagnosed severe optic neuritis. In accordance with these functional data, steroid therapy aggravated RGC apoptosis when compared with placebo-treated animals. The mechanisms of these serious unwanted steroid side effects were identified as nongenomic. In a relevant disease model of autoimmune CNS inflammation, we show for the first time that glucocorticosteroid treatment promotes neuronal cell death by a calcium-dependent inhibition of MAPK phosphorylation, a pathway involved in endogenous cell rescue.

The presented data seem to contradict recent reports of protective effects of methylprednisolone pulse therapy against whole-brain atrophy and the development of magnetic resonance imaging (MRI)-T1 black holes in MS patients (Zivadinov et al., 2001). However, treatment studies providing evidence for beneficial effects of corticosteroids were predominantly performed on patients with relapsing-remitting MS. The corticosteroid-induced enhancement of neuronal apoptosis as well as the lack of functional benefit after methylprednisolone therapy probably depend on the subtype of EAE or MS pathology. The pathogenesis of MOG-induced EAE involves the production of autoantibodies against myelin or axonal components (Stefferl et al., 1999) and early axonal and neuronal damage (Meyer et al., 2001). Disease course of MOG-EAE in our model was chronic over evaluation periods of up to $21 \mathrm{~d}$ after disease onset, and $80 \%$ of animals showed the histopathological pattern of neuromyelitis optica (data not shown). Comparing these aspects with those of the human disease, primary progressive MS (PPMS), the clinical MS subtype that barely responds to antiinflammatory and immunosuppressive treatment (Hohol et al., 1999), shows similarities in pathogenesis, pathology, and clinical appearance: As a consequence of axon degeneration and neuronal degeneration, atrophy appears to be most prominent in PPMS and often includes the spinal cord (Fox et al., 2000). Levels of autoantibodies were found to be higher in PPMS than in other MS subgroups (Sadatipour et al., 1998; Silber et al., 2002). As an additional support of the hypothesis of subgroup-specific glucocorticoid effects, methylprednisolone did not limit ongoing lesion lengthening or improve final visual outcome in patients with a progressive form of MRI-documented ON lesions triggered by an episode of acute optic neuritis (Kapoor et al., 1998).

In the present study, proapoptotic methylprednisolone effects on RGCs were mediated through a nongenomic mechanism, which can be concluded from the inability of RU 486, the classical inhibitor of the cytosolic glucocorticoid receptor (Cadepond et al., 1997), to inhibit these actions. In previous in vivo studies in rats, it has been shown that intraperitoneal delivery of RU 486 in similar concentrations as used in our experimental paradigm inhibited genomic glucocorticosteroid actions (Tjandra et al., 1996). If RU 486 was administered simultaneously with the agonist, glucocorticosteroid receptor blockade was complete because of the high receptor affinity of the steroid analog (Alexandrova, 1992). Our observation that RU 486 alone did not change RGC counts when compared with control animals showed that endogenous glucocorticosteroids produced in concentrations not sufficient for nongenomic actions had no influence on RGC survival. The effectiveness of an exogenous high-dose steroid regimen in our model can be interpreted as an additional indirect indication for nongenomic methylprednisolone effects. Non- 
genomic effects have only been described for drug concentrations far greater than the ones necessary for classical receptor saturation (Buttgereit et al., 1999). In a study comparing the effects of different glucocorticoids on rat thymocytes, it has been shown that especially methylprednisolone has a high drug potency for nonspecific nongenomic effects, such as modifying calcium cycling across the plasma membrane and thereby changing intracellular free calcium concentrations (Buttgereit et al., 1999). In the present study, cotreatment with methylprednisolone and $\mathrm{CoCl}_{2}$ revealed the functional relevance of methylprednisoloneinduced calcium influx for the suppression of MAPK phosphorylation and consecutive RGC apoptosis. Influences of intracellular calcium concentrations on phosphorylation or inactivation of MAPKs have been demonstrated for different cell types such as PC12 cells, neurons, or fibroblasts. Whereas an increase of intracellular calcium can activate MAPKs in many cells, for some cell types, opposite effects of calcium-calmodulin signaling on the activation of the MAPK pathway have been described (Agell et al., 2002).

Intracellular cascades involving MAPK phosphorylation play a crucial role in the transduction of a neurotrophic signal from the cell surface to the nucleus and are implicated in neuronal survival (Yamada et al., 2001). In different neuronal cell types, phospho-MAPK levels are increased during exposure to chronic stress, brain injury, or development of neurodegenerative diseases (Ferrer et al., 2001; Dash et al., 2002; Trentani et al., 2002). In our experimental setting, proapoptotic methylprednisolone effects were mimicked by PD 98059, a selective MEK inhibitor, indicating the functional significance of this step of the steroiddependent signal transduction cascade as well. In a previous study, it has been shown that pharmacological suppression of MAPK phosphorylation via inhibition of MEK reduced neuronal survival by mechanisms such as decreasing the ability to phosphorylate and thereby inactivating the proapoptotic protein Bad (Jin et al., 2002). Methylprednisolone exerts proapoptotic effects on RGCs by inhibiting a neuroprotective pathway that acts as a common endogenous rescue mechanism under neurodegenerative conditions. Thus, the neurodegenerative aspect of MS pathology seems to be an imperative preconditional factor for serious unwanted side effects of corticosteroid treatment. This theory was supported by our observation that healthy BN rats treated with methylprednisolone did not show any decrease in the number of surviving RGCs. Whereas glucocorticoid-induced neurodegeneration of formerly healthy neurons is regarded as specific to the hippocampus (De Kloet et al., 1998; Almeida et al., 2000), it has been shown that these drugs increase acute ischemic damage to the neocortex in rats (Tsubota et al., 1999) or augment effects of excitotoxic exposure on rat forebrain neurons (Supko and Johnston, 1994). From these results, it can be concluded that glucocorticosteroids endanger neurons, which are resistant against steroid toxicity under physiological conditions, by enhancing their vulnerability to different neurodegenerative stimuli. As a clinical parallel supporting this hypothesis, it has been shown that patients with Alzheimer's or Parkinson's disease exhibit significantly higher plasma cortisol concentrations compared with those of healthy individuals (Hartmann et al., 1997; Weiner et al., 1997), and these increased glucocorticoid levels correlate well with brain atrophy and progressive mental deterioration (De Leon et al., 1988).

In summary, we present evidence for an exacerbation of neuronal apoptosis under methylprednisolone treatment in an animal model that especially reflects neurodegenerative aspects of MS. The data presented suggest that there may be subgroups of patients with chronic inflammatory autoimmune CNS disease who are endangered by unwanted side effects of high-dose methylprednisolone therapy that could promote ongoing neuronal degeneration. Furthermore, these results suggest that combination therapies targeting both inflammatory and neurodegenerative aspects of MS need to be developed in the future.

\section{References}

Agell N, Bachs O, Rocamora N, Villalonga P (2002) Modulation of the Ras/ Raf/MEK/ERK pathway by $\mathrm{Ca}^{2+}$, and calmodulin. Cell Signal 14:649-654.

Alexandrova M (1992) Duration of antagonizing effect of RU 486 on the agonist induction of tyrosine aminotransferase via glucocorticoid receptor. J Steroid Biochem Mol Biol 41:723-725.

Almeida OF, Conde GL, Crochemore C, Demeneix BA, Fischer D, Hassan AH, Meyer M, Holsboer F, Michaelidis TM (2000) Subtle shifts in the ratio between pro- and antiapoptotic molecules after activation of corticosteroid receptors decide neuronal fate. FASEB J 14:779-790.

Beck RW, Cleary PA, Anderson Jr MM, Keltner JL, Shults WT, Kaufman DI, Buckley EG, Corbett JJ, Kupersmith MJ, Miller NR, Savino PJ, Guy R, Trobe JD, McCrary JA, Smith CH, Chrousos GA, Thompson HS, Katz BJ, Brodsky MC, Goodwin JA, et al. (1992) A randomized, controlled trial of corticosteroids in the treatment of acute optic neuritis. The Optic Neuritis Study Group. N Engl J Med 326:581-588.

Brusaferri F, Candelise L (2000) Steroids for multiple sclerosis and optic neuritis: a meta-analysis of randomized controlled clinical trials. J Neurol 247:435-442.

Buttgereit F, Brand MD, Burmester GR (1999) Equivalent doses and relative drug potencies for nongenomic glucocorticoid effects: a novel glucocorticoid hierachy. Biochem Pharmacol 58:363-368.

Cadepond F, Ulmann A, Baulieu EE (1997) RU 486 (mifepristone): mechanisms of action and clinical uses. Annu Rev Med 48:129-156.

Dash PK, Mach SA, Moore AN (2002) The role of extracellular signalregulated kinase in cognitive and motor deficits following experimental traumatic brain injury. Neuroscience 114:755-767.

De Kloet ER, Vreugdenhil E, Oitzl MS, Joels M (1998) Brain corticosteroid receptor balance in health and disease. Endocr Rev 19:269-301.

De Leon MJ, McRae T, Tsai JR, George AE, Marcus DL, Freedman M, Wolf AP, McEwen BS (1988) Abnormal cortisol response in Alzheimer's disease linked to hippocampal atrophy. Lancet 2:391-392.

Diem R, Meyer R, Weishaupt JH, Bähr M (2001) Reduction of potassium currents and phosphatidylinositol 3-kinase-dependent Akt phosphorylation by tumor necrosis factor- $\alpha$ rescues axotomized retinal ganglion cells from retrograde cell death in vivo. J Neurosci 21:2058-2066.

Dorscheid DR, Wojcik KR, Sun S, Marroquin B, White SR (2001) Apoptosis of airway epithelial cells induced by corticosteroids. Am J Respir Crit Care Med 164:1939-1947.

Falkenstein E, Tillmann HC, Christ M, Feuring M, Wehling M (2000) Multiple actions of steroid hormones: a focus on rapid, nongenomic effects. Pharmacol Rev 52:513-556.

Ferrer I, Blanco R, Carmona M, Ribera R, Goutan E, Puig B, Rey MJ, Cardozo A, Vinals F, Ribalta T (2001) Phosphorylated map kinase (ERK1, ERK2) expression is associated with early tau deposition in neurones and glial cells, but not with increased nuclear DNA vulnerability and cell death, in Alzheimer disease, Pick's disease, progressive supranuclear palsy and corticobasal degeneration. Brain Pathol 11:144-158.

Fox NC, Jenkins R, Leary SM, Stevenson VL, Losseff NA, Crum WR, Harvey RJ, Rossor MN, Miller DH, Thompson AJ (2000) Progressive cerebral atrophy in MS. A serial study using registered volumetric MRI. Neurology 54:807-812.

Gelati M, Corsini E, De Rossi M, Masini L, Bernardi G, Massa G, Boiardi A, Salmaggi A (2002) Methylprednisolone acts on peripheral blood mononuclear cells and endothelium in inhibiting migration phenomena in patients with multiple sclerosis. Arch Neurol 59:774-780.

Gold R, Buttgereit F, Toyka KV (2001) Mechanism of action of glucocorticosteroid hormones: possible implications for therapy of neuroimmunological disorders. J Neuroimmunol 117:1-8.

Hartmann A, Veldhuis JD, Deuschle M, Standhardt H, Heuser I (1997) Twenty-four hour cortisol release profiles in patients with Alzheimer's and Parkinson's disease compared to normal controls: ultradian secretory pulsatility and diurnal variation. Neurobiol Aging 18:285-289.

Hohol MJ, Olek MJ, Orav EJ, Stazzone L, Hafler DA, Khoury SJ, Dawson DM, 
Weiner HL (1999) Treatment of progressive multiple sclerosis with pulse cyclophosphamide/methylprednisolone: response to therapy is linked to the duration of progressive disease. Mult Scler 5:403-409.

Jin K, Mao XO, Zhu Y, Greenberg DA (2002) MEK and ERK protect hypoxic cortical neurons via phosphorylation of Bad. J Neurochem 80:119-125.

Kapoor R, Miller DH, Jones SJ, Plant GT, Brusa A, Gass A, Hawkins CP, Page R, Wood NW, Compston DA, Moseley IF, McDonald WI (1998) Effects of intravenous methylprednisolone on outcome in MRI-based prognostic subgroups in acute optic neuritis. Neurology 50:230-237.

Kaufman DI, Trobe JD, Eggenberger ER, Whitaker JN (2000) Practice parameter: the role of corticosteroids in the management of acute monosymptomatic optic neuritis-report of the Quality Standards Subcommitee of the American Academy of Neurology. Neurology 54:2039-2044.

Kültz D, Madhany S, Burg MB (1998) Hyperosmolality causes growth arrest of murine kidney cells. Induction of GADD45 and GADD153 by osmosensing via stress-activated protein kinase 2. J Biol Chem 273:13645-13651.

Leussink VI, Jung S, Merschdorf U, Toyka KV, Gold R (2001) High-dose methylprednisolone therapy in multiple sclerosis induces apoptosis in peripheral blood leukocytes. Arch Neurol 58:91-97.

Meyer R, Weissert R, Diem R, Storch MK, de Graaf KL, Kramer B, Bähr M (2001) Acute neuronal apoptosis in a rat model of multiple sclerosis. J Neurosci 21:6214-6220.

Nakazawa F, Matsuno H, Yudoh K, Watanabe Y, Katayama R, Kimura T (2002) Corticosteroid treatment induces chondrocyte apoptosis in an experimental arthritis model and in chondrocyte cultures. Clin Exp Rheumatol 20:773-781.

Sadatipour BT, Greer JM, Pender MP (1998) Increased circulating antiganglioside antibodies in primary and secondary progressive multiple sclerosis. Ann Neurol 44:980-983.

Schenberg LC, Marcal LP, Seeberger F, Barros MR, Sudre EC (2000) L-type calcium channels selectively control the defensive behaviors induced by electrical stimulation of dorsal periaqueductal gray and overlying collicular layers. Behav Brain Res 111:175-185.

Schmidt J, Gold R, Schönrock L, Zettl UK, Hartung HP, Toyka KV (2000) T-cell apoptosis in situ in experimental autoimmune encephalomyelitis following methylprednisolone pulse therapy. Brain 123:1431-1441.

Silber E, Semra YK, Gregson NA, Sharief MK (2002) Patients with progressive multiple sclerosis have elevated antibodies to neurofilament subunit. Neurology 58:1372-1381.

Stefferl A, Brehm U, Storch MK, Lambracht-Washington D, Bourquin C, Wonigeit K, Lassmann H, Linington C (1999) Myelin oligodendrocyte glycoprotein induces experimental autoimmune encephalomyelitis in the "resistant" Brown Norway rat: disease susceptibility is determined by MHC and MHC-linked effects on the B cell response. J Immunol 163:40-49.

Storch MK, Stefferl A, Brehm U, Weissert R, Wallstrom E, Kerschensteiner M, Olsson T, Linington C, Lassmann H (1998) Autoimmunity to myelin oligodendrocyte glycoprotein in rats mimics the spectrum of multiple sclerosis pathology. Brain Pathol 8:681-694.

Supko D, Johnston M (1994) Dexamethasone potentiates NMDA receptormediated neuronal injury in the postnatal rat. Eur J Pharmacol 270:105-109.

Sze PY, Iqbal Z (1994) Regulation of calmodulin content in synaptic plasma membranes by glucocorticoids. Neurochem Res 19:1455-1461.

Tjandra K, Kubes P, Rioux K, Swain MG (1996) Endogenous glucocorticoids inhibit neutrophil recruitment to inflammatory sites in cholestatic rats. Am J Physiol 270:G821-G825.

Trentani A, Kuipers SD, Ter Horst GJ, Den Boer JA (2002) Selective chronic stress-induced in vivo ERK1/2 hyperphosphorylation in medial prefrontocortical dendrites: implications for stress-related cortical pathology? Eur J Neurosci 15:1681-1691.

Tsubota S, Adachi N, Chen J, Yorozuya T, Nagaro T, Arai T (1999) Dexamethasone changes brain monoamine metabolism and aggravates ischemic neuronal damage in rats. Anesthesiology 90:515-523.

Venero C, Borrell J (1999) Rapid glucocorticoid effects on excitatory amino acid levels in the hippocampus: a microdialysis study in freely moving rats. Eur J Neurosci 11:2465-2473.

Wandinger KP, Wessel K, Trillenberg P, Heindl N, Kirchner H (1998) Effect of high-dose methylprednisolone administration on immune functions in multiple sclerosis patients. Acta Neurol Scand 97:359-365.

Watters JJ, Campbell JS, Cunningham MJ, Krebs EG, Dorsa DM (1997) Rapid membrane effects of steroids in neuroblastoma cells: effects of estrogen on mitogen activated protein kinase signalling cascade and c-fos immediate early gene transcription. Endocrinology 138:4030-4033.

Weiner MF, Vobach S, Olsson K, Svetlik D, Risser RC (1997) Cortisol secretion and Alzheimer's disease. Biol Psychiatry 42:1030-1038.

Weissert R, Wallstrom E, Storch MK, Stefferl A, Lorentzen J, Lassmann H, Linington C, Olsson T (1998) MHC haplotype-dependent regulation of MOG-induced EAE in rats. J Clin Invest 102:1265-1273.

Yamada M, Tanabe K, Wada K, Shimoke K, Ishikawa Y, Ikeuchi T, Koizumi S, Hatanaka H (2001) Differences in survival-promoting effects and intracellular signaling properties of BDNF and IGF-1 in cultured cerebral cortical neurons. J Neurochem 78:940-951.

Zivadinov R, Rudick RA, De Masi R, Nasuelli D, Ukmar M, Pozzi-Mucelli RS, Grop A, Cazzato G, Zorzon M (2001) Effects of IV methylprednisolone on brain atrophy in relapsing-remitting MS. Neurology 57:1239-1247. 